\title{
Prospective Associations Between Alcohol Consumption and Psychological Well-being in Midlife
}

\author{
Marie Grønkjær ( $\square$ marie.stjerne.groenkjaer@regionh.dk ) \\ Bispebjerg and Frederiksberg Hospital https://orcid.org/0000-0002-7049-0952 \\ Cathrine Lawaetz Wimmelmann \\ University of Copenhagen Department of Public Health: Kobenhavns Universitet Institut for Folkesundhedsvidenskab \\ Erik Lykke Mortensen \\ University of Copenhagen Department of Public Health: Kobenhavns Universitet Institut for Folkesundhedsvidenskab \\ Trine Flensborg-Madsen \\ University of Copenhagen Department of Public Health: Kobenhavns Universitet Institut for Folkesundhedsvidenskab
}

\section{Research article}

Keywords: Psychological well-being, quality of life, satisfaction with life, vitality, alcohol consumption, binge drinking

Posted Date: July 28th, 2021

DOI: https://doi.org/10.21203/rs.3.rs-743633/v1

License: (a) (7) This work is licensed under a Creative Commons Attribution 4.0 International License. Read Full License

Version of Record: A version of this preprint was published at BMC Public Health on January 31st, 2022. See the published version at https://doi.org/10.1186/s12889-021-12463-4. 


\section{Abstract}

Background: Alcohol consumption potentially influences psychological well-being in beneficial and harmful ways, but prospective studies on the association show mixed results. Our main purpose was to examine prospective associations between alcohol consumption and psychological well-being in middle-aged men and women.

Methods: The study sample included 4,148 middle-aged individuals ( $80 \%$ men) from the Copenhagen Aging and Midlife Biobank who reported their alcohol consumption (average weekly consumption and frequency of binge drinking) at baseline in 2004 or 2006 and reported their psychological well-being (satisfaction with life and vitality) at follow-up in 2009-2011. Analyses were adjusted for sociodemographic factors, lifestyle, social relations, and morbidity.

Results: For satisfaction with life at follow-up, lower scores were observed in men and women who were alcohol abstinent at baseline as well as in men with heavy alcohol consumption compared with moderate alcohol consumption at baseline. Moreover, men with moderate frequency of binge drinking (1-3 times/month) at baseline had higher satisfaction with life scores at follow-up than men with less and more frequent binge drinking. In relation to vitality at follow-up, alcohol abstinence at baseline in men and women and heavy alcohol consumption at baseline in men were associated with lower scores compared with moderate alcohol consumption.

Conclusions: Alcohol abstinence seems to be prospectively associated with adverse psychological well-being in men and women, while heavy alcohol consumption seems to be prospectively associated with adverse psychological well-being in men. Finally, an inverse ushaped prospective association between binge drinking and life satisfaction was observed in men.

\section{Background}

The influence of alcohol consumption on psychological well-being is complex and remains a debated topic. In the short-term, alcohol may be associated with pleasure and positive effects such as stress reduction, having fun and letting go of control. Nevertheless, alcohol may also lead to acute negative outcomes such as intoxication. In the long-term, studies have suggested that alcohol may have both beneficial and harmful effects on psychological well-being. Cross-sectional studies have suggested inversely J-shaped associations between alcohol consumption and psychological well-being in terms of low psychological well-being with heavy alcohol consumption and frequent binge drinking [1-4] and alcohol abstinence [4-8]. Some prospective studies likewise suggest that heavy alcohol consumption and frequent binge drinking are associated with lower psychological well-being [9-11] and that alcohol abstinence is associated with lower psychological well-being than moderate consumption [10,12-14]. However, some studies have also implied that alcohol consumption is not prospectively associated with psychological well-being [15-17]. One study has even suggested that larger alcohol consumption among university students was associated with higher subjective well-being two years later [18].

The conflicting evidence on the prospective association between alcohol consumption and psychological well-being may partly be caused by diverse study samples and differences in assessment of alcohol consumption and psychological well-being. For example, age and sex differences in the association have previously been suggested $[10,19]$. Anticipated age differences underline the importance of investigating the association in different age groups, such as in middle-aged and older individuals $[11-13,16]$. Since alcohol consumption may influence different aspects of psychological well-being in different ways, it is problematic that existing studies in middle-aged and older participants have primarily focused on health-related quality of life. Finally, the consumption pattern may be important in relation to psychological well-being but there is scarcity of studies examining binge drinking and psychological well-being in midlife and old age.

The purpose of the present study was to examine prospective associations between alcohol consumption and psychological well-being in middle-aged men and women in Denmark. More specifically, the influence of both average weekly alcohol consumption and frequency of binge drinking was investigated in relation to two aspects of psychological well-being comprising satisfaction with life and vitality.

\section{Methods}

\section{Study participants}

The study population consisted of participants from the Copenhagen Aging and Midlife Biobank (CAMB) [20]. CAMB is a follow-up study comprising participants from three established cohorts; in the present study, we included men from the Metropolit 1953 Danish male birth cohort (MP) [21] and men and women from the Danish Longitudinal Study on Work, Unemployment and Health (DALWUH) [22]. The MP cohort included all boys born in 1953 in the Copenhagen Metropolitan area $(N=12,270)$ [21], and 6,219 of these boys completed a questionnaire in 2004 [23]. The DALWUH cohort included a random sample of Danish men and women aged 40 or 50 in $1999(\mathrm{~N}=11,082)$ [20], and 6,151 of these individuals completed a questionnaire in 2006 [23]. 
From the original cohorts, 7,750 from the MP cohort and 4,906 from the DALWUH cohort were invited to participate in the CAMB study, which included a questionnaire and clinical tests. All participants gave informed consent [20]. In total, 4160 individuals (2,749 from MP and 1,411 from DALWUH) completed the questionnaire both at baseline in 2004 (MP) or 2006 (DALWUH) and at follow-up in $2009-2011$ (CAMB). However, 4148 individuals were included in the analyses because 12 individuals were excluded to avoid cells with less than three observations.

Alcohol consumption at baseline

\section{Average alcohol consumption}

MP cohort members self-reported the amount of alcohol consumed the past week in terms of bottles of regular and strong beer, glasses of red and white wine, glasses of fortified wine, and units of spirits. Using the same alcohol categories, DALWUH cohort members selfreported the amount of alcohol consumed on typical weekdays and in typical weekends.

This information was used to calculate average weekly units of alcohol consumed using the following equivalents: regular beer (bottle) = 1 unit; strong beer (bottle) = 2 units; red wine (glass) = 1 unit; white wine (glass) = 1 unit; fortified wine (glass) = 11\%2 units; and spirits reported in units. In Denmark and in this study, one unit of alcohol corresponds to 12 grams of pure alcohol.

\section{Binge drinking}

MP cohort members were asked to report the number of times that they had consumed $\geq 5$ units of alcohol on the same occasion during the last 30 days. A categorical binge drinking variable was constructed with three categories: 'less than once a month'; '1-3 times per month'; and 'once per week or more'. DALWUH cohort members were not asked questions about binge drinking.

Psychological well-being at follow-up

\section{Satisfaction with life}

Participants' life satisfaction was assessed by the Satisfaction With Life Scale (SWLS) [24]. Five general statements related to the respondents' life satisfaction are included in SWLS. The five general statements are answered using a 7-point scale ranging from 1 (strongly disagree) to 7 (strongly agree), leading to a total sum-score ranging from 5 to 35 . The psychometric properties of SWLS in terms of both reliability and validity have shown to be satisfactory $[24,25]$. In the present study sample, Cronbach's alpha for the five SWLS items was 0.91 .

\section{Vitality}

Participants' vitality was assessed by the vitality scale of the Medical Outcomes Study 36-Item Short-Form Health Survey (SF-36) [26] at both baseline and follow-up. Four items are included in SF-36 to evaluate participants' vitality by referring to feelings of energy and fatigue within the past month, for example "How much of the time during the past 4 weeks did you have a lot of energy?". Scores were

transformed to a continuous scale ranging from 0 to 100 with higher scores indicating higher vitality [27]. Investigation of the psychometric properties of the Danish translation of the vitality scale has indicated that both the reliability and validity is satisfactory [28, 29]. In the present study sample, Cronbach's alpha for the four SF-36 vitality items was 0.88 at both baseline and follow-up.

Covariates

Information on covariates was obtained from the questionnaires completed at baseline and from the Danish National Patient Register [30] using the unique personal identification number.

\section{Sociodemographic factors}

Participants' reported the level of vocational training ranging from 'no vocational training' to 'higher level education $>4$ years' and this information was used to construct a variable indicating whether the participant's educational leve/ was low, medium, or high. Moreover, dichotomous information on current employment was included.

\section{Other lifestyle factors}

Smoking was included in three categories: never-smoker; ex-smoker; and current smoker. Information on leisure time physical activity (assessed differently in the two cohorts) was used to construct three groups: moderate/heavy activity (moderate or heavy exercise $\geq 4$ hours/week or $\geq 7$ hours of physical activity); light activity (light exercise $\geq 4$ hours/week or 2-6 hours of physical activity); and sedentary (sedentary leisure activities or $<2$ hours of physical activity/week). Body Mass Index (BMI) was grouped into $<30$ vs. $\geq 30$ kg/m². 


\section{Social relations}

Three dichotomized variables on social relations were included: children (yes/no; including biological, adopted, and foster children); living alone (yes/no); and a variable, which indicated whether participants rarely/never had someone to talk well with (yes/no)(neither family, friends, partner, children nor colleagues).

\section{Morbidity}

Self-rated health was reported on a 5-point scale from 1 (poor) to 5 (excellent) and grouped into low (1), medium (2-3) and high (4-5). Charlson Comorbidity Index (CCl) was calculated using information from the Danish National Patient Register from 1977 to 2003 . CCl is a method of measuring somatic comorbidity by weighting different diseases based on the influence on mortality [31]. The updated weights suggested by Quan et al. [32] were used and three groups were constructed based on the scores: 0,1 , and $\geq 2$. Using information from the Danish National Patient Register on hospital registrations from psychiatric wards from 1994 to 2003, a dichotomous variable was generated to indicate whether participants had psychiatric hospital diagnoses (ICD-10: F10-F99).

\section{Statistical methods}

Characteristics of the study sample split on weekly units of alcohol at baseline were examined and between-group differences were analyzed using $\chi^{2}$ test for categorical and one-way ANOVA for continuous variables (Table 1 ). The missing data frequency was $\leq 3 \%$ for all variables, except for weekly alcohol consumption (3.5\%), and SF-36 vitality scores from both baseline (5.5\%) and follow-up (3.8\%). Cross-sectional associations between alcohol consumption and vitality scores at baseline were investigated and tested using one-way ANOVA (Table 2). Separate associations between the two alcohol consumption variables-average weekly consumption and frequency of binge drinking-at baseline and psychological well-being at follow-up were examined by Full Information Maximum Likelihood models in Stata's SEM package to handle missing data. Results from unadjusted analyses and from four adjusted models were presented. The first adjusted model included adjustment for sociodemographic factors including age, cohort, education, and employment (Model 1). These adjustment factors were also included in the remainder of the adjusted models that included further adjustment for 2. other lifestyle factors, 3. social relations, and 4. morbidity. Results with SWLS score and SF-36 vitality score as the outcome are presented in Table 3 and Table 4, respectively. Sensitivity analyses were completed using only participants with complete information on all included variables, revealing highly similar results. All analyses were conducted using Stata version 14.2. 
Table 1

Characteristics of the study sample $(\mathrm{N}=4148)$

\begin{tabular}{|c|c|c|c|c|c|c|}
\hline & \multirow[t]{2}{*}{ Total } & \multicolumn{5}{|c|}{ Weekly units of alcohol at baseline } \\
\hline & & 0 units & $1-14$ units & $15-21$ units & $\geq 22$ units & p-value ${ }^{a}$ \\
\hline Total & $N=4003$ & $280(7.0)$ & $2136(53.4)$ & $670(16.7)$ & $917(22.9)$ & \\
\hline \multicolumn{7}{|l|}{ Sociodemographic factors } \\
\hline Age (mean [SD]) & $51.7(3.0)$ & $51.5(3.5)$ & $51.6(3.3)$ & $51.6(2.6)$ & $51.6(2.5)$ & 0.821 \\
\hline $\operatorname{Sex}(N[\%])$ & & & & & & $<0.001$ \\
\hline Male & $3336(80.4)$ & $183(65.4)$ & $1592(74.5)$ & 594 (88.7) & 860 (93.8) & \\
\hline Female & $812(19.6)$ & $97(34.6)$ & $544(25.5)$ & $76(11.3)$ & $57(6.2)$ & \\
\hline Cohort (N [\%]) & & & & & & $<0.001$ \\
\hline MP cohort & $2746(66.2)$ & $148(52.9)$ & $1282(60.0)$ & $512(76.4)$ & $727(79.3)$ & \\
\hline DALWUH cohort & $1402(33.8)$ & $132(47.1)$ & $854(40.0)$ & $158(23.6)$ & $190(20.7)$ & \\
\hline Educational level (N [\%]) & & & & & & $<0.001$ \\
\hline Low & $527(12.8)$ & $67(23.9)$ & $234(11.0)$ & $73(10.9)$ & $115(12.6)$ & \\
\hline Medium & 1949 (47.3) & $127(45.4)$ & $1006(47.5)$ & $306(45.7)$ & $441(48.2)$ & \\
\hline High & 1643 (39.9) & $86(30.7)$ & 879 (41.5) & $291(43.4)$ & 358 (39.2) & \\
\hline Currently employed (N [\%]) & & & & & & $<0.001$ \\
\hline Yes & $3782(91.5)$ & $214(76.4)$ & $1994(93.7)$ & $634(94.6)$ & 835 (91.1) & \\
\hline No & $353(8.5)$ & $66(23.6)$ & $134(6.3)$ & $36(5.4)$ & $82(8.9)$ & \\
\hline \multicolumn{7}{|l|}{ Other lifestyle factors } \\
\hline Smoking (N [\%]) & & & & & & $<0.001$ \\
\hline Current smoker & $1282(31.4)$ & $112(40.0)$ & $534(25.0)$ & $188(28.1)$ & $403(44.1)$ & \\
\hline Ex-smoker & $1438(35.1)$ & $88(31.4)$ & $750(35.2)$ & $274(40.9)$ & $302(33.0)$ & \\
\hline Never-smoker & 1370 (33.5) & $80(28.6)$ & $848(39.8)$ & $208(31.0)$ & 209 (22.9) & \\
\hline Leisure time physical activity (N [\%]) & & & & & & $<0.001$ \\
\hline Heavy/moderate exercise & $1219(29.9)$ & $81(28.9)$ & $671(31.6)$ & $211(31.5)$ & $231(25.3)$ & \\
\hline Light exercise & $2343(57.4)$ & $146(52.1)$ & $1202(56.6)$ & $384(57.3)$ & $559(61.2)$ & \\
\hline Sedentary activities & $521(12.8)$ & $53(18.9)$ & $250(11.8)$ & $75(11.2)$ & $124(13.6)$ & \\
\hline Body Mass Index (N [\%]) & & & & & & 0.002 \\
\hline$<30 \mathrm{~kg} / \mathrm{m}^{2}$ & 3641 (89.4) & $227(84.1)$ & $1890(89.8)$ & $606(92.4)$ & 805 (89.2) & \\
\hline$\geq 30 \mathrm{~kg} / \mathrm{m}^{2}$ & $431(10.6)$ & $43(15.9)$ & $214(10.2)$ & $50(7.6)$ & $97(10.8)$ & \\
\hline \multicolumn{7}{|l|}{ Social relations } \\
\hline Children (N [\%]) & & & & & & $<0.001$ \\
\hline Yes & $3504(84.8)$ & $225(80.4)$ & $1860(87.5)$ & $559(83.4)$ & $752(82.0)$ & \\
\hline No & $626(15.2)$ & $55(19.6)$ & 265 (12.5) & $111(16.6)$ & $165(18.0)$ & \\
\hline Living alone (N [\%]) & & & & & & $<0.001$ \\
\hline Yes & $640(15.5)$ & $79(28.2)$ & $278(13.1)$ & $74(11.1)$ & $161(17.7)$ & \\
\hline
\end{tabular}




\begin{tabular}{|c|c|c|c|c|c|c|}
\hline & \multirow[t]{2}{*}{ Total } & \multicolumn{5}{|c|}{ Weekly units of alcohol at baseline } \\
\hline & & 0 units & $1-14$ units & $15-21$ units & $\geq 22$ units & p-value ${ }^{a}$ \\
\hline No & $3478(84.5)$ & $201(71.8)$ & $1844(86.9)$ & $591(88.9)$ & $750(82.3)$ & \\
\hline Rarely have someone to talk well with (N [\%]) & & & & & & 0.062 \\
\hline Yes & $35(0.9)$ & $6(2.1)$ & $15(0.7)$ & $4(0.6)$ & $6(0.7)$ & \\
\hline No & $4081(99.2)$ & $274(97.9)$ & $2108(99.3)$ & $662(99.4)$ & $903(99.3)$ & \\
\hline \multicolumn{7}{|l|}{ Morbidity } \\
\hline Self-rated health (N [\%]) & & & & & & $<0.001$ \\
\hline Low & $399(9.7)$ & $67(24.2)$ & $159(7.5)$ & $42(6.3)$ & $87(9.6)$ & \\
\hline Medium & $3180(77.6)$ & $184(66.4)$ & $1668(79.0)$ & $532(80.2)$ & $718(79.2)$ & \\
\hline High & $518(12.6)$ & $26(9.4)$ & $285(13.5)$ & $89(13.4)$ & $101(11.1)$ & \\
\hline Charlson Comorbidity Index score (N [\%]) & & & & & & 0.005 \\
\hline 0 & $3880(93.5)$ & $252(90.0)$ & $2017(94.4)$ & $640(95.5)$ & $853(93.0)$ & \\
\hline 1 & $136(3.3)$ & $14(5.0)$ & $54(2.5)$ & $17(2.5)$ & $40(4.4)$ & \\
\hline$\geq 2$ & $132(3.2)$ & $14(5.0)$ & $65(3.0)$ & $13(1.9)$ & $24(2.6)$ & \\
\hline Psychiatric hospital diagnoses (N [\%]) & & & & & & $<0.001$ \\
\hline Yes & $131(3.2)$ & $26(9.3)$ & $40(1.9)$ & $13(1.9)$ & $27(2.9)$ & \\
\hline No & $4017(96.8)$ & $254(90.7)$ & $2096(98.1)$ & $657(98.1)$ & $890(97.1)$ & \\
\hline \multicolumn{7}{|l|}{ Alcohol consumption (exposure variable) } \\
\hline Binge drinking $(\mathrm{N}[\%])^{b}$ & & & & & & $<0.001$ \\
\hline$<$ once per month & $712(26.7)$ & $106(71.6)$ & $507(40.0)$ & $55(10.8)$ & $27(3.8)$ & \\
\hline $1-3$ times per month & $1095(41.1)$ & $37(25.0)$ & $628(49.6)$ & $258(50.6)$ & $170(23.7)$ & \\
\hline$\geq$ once per week & $858(32.2)$ & $5(3.4)$ & $131(10.3)$ & $197(38.6)$ & $521(72.6)$ & \\
\hline \multicolumn{6}{|l|}{1 unit corresponds to 12 grams of pure alcohol } & ${ }^{a} \chi^{2}$ test for categorical and one-way ANOVA for continuous variables \\
\hline \multicolumn{7}{|c|}{$\begin{array}{l}{ }^{b} \text { Only including men from the Metropolit } 1953 \text { Danish male birth cohort }(N=2746) \text { as information on binge drinking was not available } \\
\text { in the Danish Longitudinal Study on Work, Unemployment and Health cohort }\end{array}$} \\
\hline
\end{tabular}


Table 2

SF-36 vitality scores at baseline by alcohol consumption at baseline

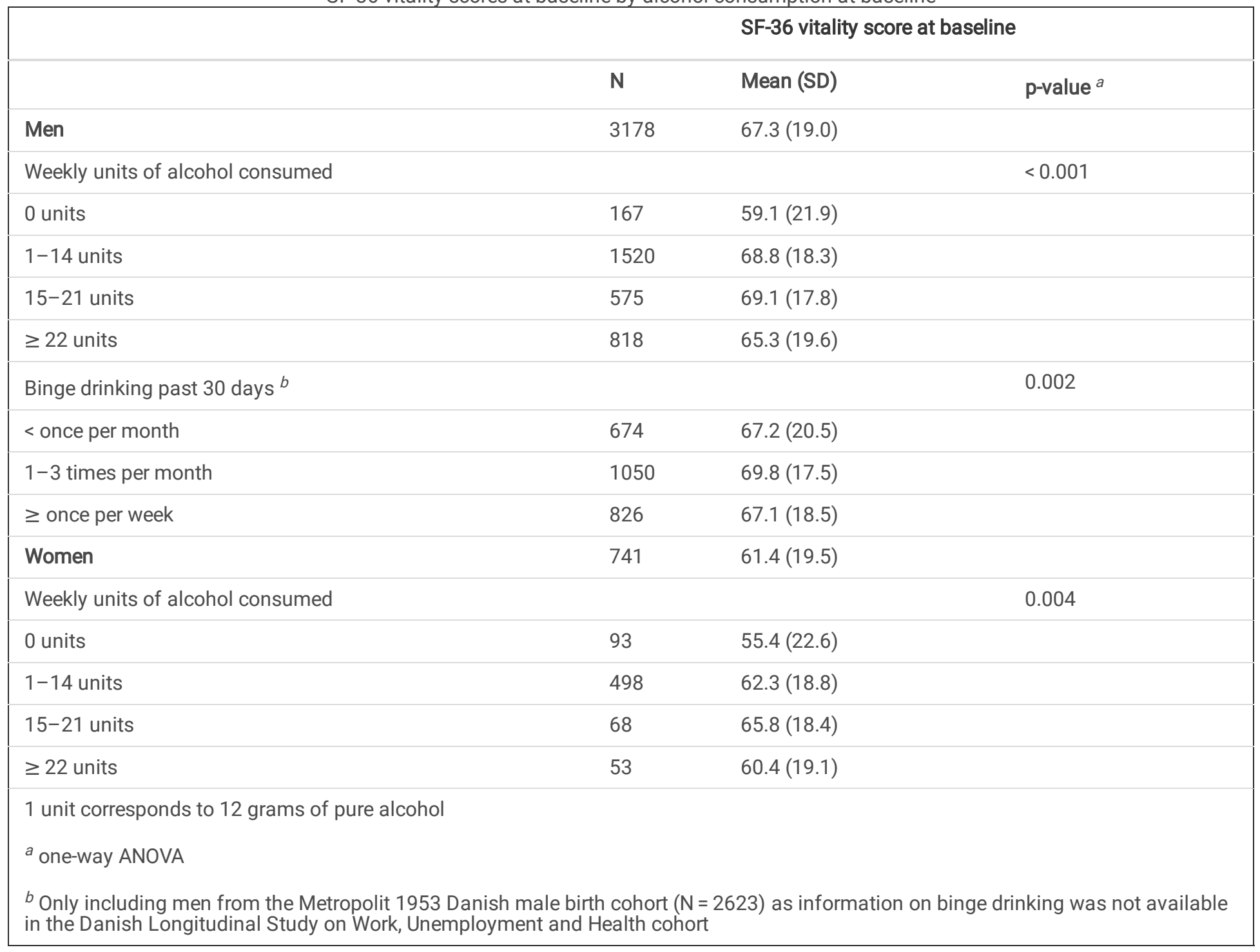


Table 3

FIML analyses on associations between alcohol consumption at baseline and SWLS score at follow-up in unadjusted and adjusted SEM models in men $(\mathrm{N}=3336)$ and women $(\mathrm{N}=812)$

\begin{tabular}{|c|c|c|c|c|c|c|c|c|c|c|}
\hline \multirow[b]{2}{*}{$\begin{array}{l}\text { Men }(N= \\
3336)\end{array}$} & \multicolumn{2}{|l|}{ Unadjusted $^{a}$} & \multicolumn{2}{|c|}{$\begin{array}{l}\text { Model } 1 \text { (age, cohort, } \\
\text { education, } \\
\text { employment) }\end{array}$} & \multicolumn{2}{|c|}{$\begin{array}{l}\text { Model } 1 \text { + other } \\
\text { lifestyle factors }\end{array}$} & \multicolumn{2}{|c|}{$\begin{array}{l}\text { Model } 1 \text { + social } \\
\text { relations }\end{array}$} & \multicolumn{2}{|c|}{ Model 1 + morbidity } \\
\hline & $\mathrm{B}(95 \% \mathrm{Cl})$ & $\begin{array}{l}\mathrm{p}- \\
\text { value }\end{array}$ & B $(95 \% \mathrm{Cl})$ & $\begin{array}{l}\mathrm{p}- \\
\text { value }\end{array}$ & $\mathrm{B}(95 \% \mathrm{Cl})$ & $\begin{array}{l}\mathrm{p}- \\
\text { value }\end{array}$ & $\mathrm{B}(95 \% \mathrm{Cl})$ & $\begin{array}{l}\mathrm{p}- \\
\text { value }\end{array}$ & B $(95 \% \mathrm{Cl})$ & $\begin{array}{l}\mathrm{p}- \\
\text { value }\end{array}$ \\
\hline \multicolumn{11}{|l|}{$\begin{array}{l}\text { Weekly } \\
\text { units of } \\
\text { alcohol }\end{array}$} \\
\hline 0 units & $\begin{array}{l}-3.27 \\
(-4.11 ;-2.44)\end{array}$ & $<.001$ & $\begin{array}{l}-1.83 \\
(-2.64 ;-1.02)\end{array}$ & $<.001$ & $\begin{array}{l}-1.61 \\
(-2.41 ;-0.80)\end{array}$ & $<.001$ & $\begin{array}{l}-1.44 \\
(-2.24 ;-0.65)\end{array}$ & $<.001$ & $\begin{array}{l}-1.27 \\
(-2.06 ;-0.49)\end{array}$ & 0.001 \\
\hline $1-14$ units & Ref & - & Ref & - & Ref & - & Ref & - & Ref & - \\
\hline $\begin{array}{l}15-21 \\
\text { units }\end{array}$ & $\begin{array}{l}0.10 \\
(-0.41 ; 0.62)\end{array}$ & 0.693 & $\begin{array}{l}0.05 \\
(-0.43 ; 0.54)\end{array}$ & 0.828 & $\begin{array}{l}0.05 \\
(-0.44 ; 0.53)\end{array}$ & 0.855 & $\begin{array}{l}0.03 \\
(-0.44 ; 0.51)\end{array}$ & 0.887 & $\begin{array}{l}0.09 \\
(-0.38 ; 0.56)\end{array}$ & 0.713 \\
\hline$\geq 22$ units & $\begin{array}{l}-0.99 \\
(-1.45 ;-0.54)\end{array}$ & $<.001$ & $\begin{array}{l}-0.79 \\
(-1.23 ;-0.36)\end{array}$ & $\dot{0} 0.001$ & $\begin{array}{l}-0.59 \\
(-1.03 ;-0.16)\end{array}$ & 0.007 & $\begin{array}{l}-0.67 \\
(-1.09 ;-0.25)\end{array}$ & 0.002 & $\begin{array}{l}-0.63 \\
(-1.04 ;-0.22)\end{array}$ & 0.003 \\
\hline \multicolumn{11}{|l|}{$\begin{array}{l}\text { Binge } \\
\text { drinking } \\
\text { past } 30\end{array}$} \\
\hline $\begin{array}{l}<\text { once per } \\
\text { month }\end{array}$ & $\begin{array}{l}-0.97 \\
(-1.49 ;-0.45)\end{array}$ & $<.001$ & $\begin{array}{l}-0.64 \\
(-1.13 ;-0.15)\end{array}$ & 0.010 & $\begin{array}{l}-0.66 \\
(-1.15 ;-0.17)\end{array}$ & 0.008 & $\begin{array}{l}-0.46 \\
(-0.94 ; 0.02)\end{array}$ & 0.060 & $\begin{array}{l}-0.43 \\
(-0.91 ; 0.04)\end{array}$ & 0.074 \\
\hline $\begin{array}{l}1-3 \text { times } \\
\text { per month }\end{array}$ & Ref & - & Ref & - & Ref & - & Ref & - & Ref & - \\
\hline $\begin{array}{l}\geq \text { once per } \\
\text { week }\end{array}$ & $\begin{array}{l}-0.98 \\
(-1.47 ;-0.49)\end{array}$ & $\begin{array}{l}< \\
0.001\end{array}$ & $\begin{array}{l}-0.76 \\
(-1.22 ;-0.30)\end{array}$ & 0.001 & $\begin{array}{l}-0.62 \\
(-1.08 ;-0.16)\end{array}$ & 0.009 & $\begin{array}{l}-0.60 \\
(-1.05 ;-0.14)\end{array}$ & 0.010 & $\begin{array}{l}-0.72 \\
(-1.16 ;-0.27)\end{array}$ & 0.002 \\
\hline $\begin{array}{l}\text { Women }(\mathrm{N} \\
=812)\end{array}$ & B (95\% Cl) & $\begin{array}{l}\mathrm{p} \text { - } \\
\text { value }\end{array}$ & B $(95 \% \mathrm{Cl})$ & $\begin{array}{l}\mathrm{p}- \\
\text { value }\end{array}$ & $\mathrm{B}(95 \% \mathrm{Cl})$ & $\begin{array}{l}\mathrm{p}- \\
\text { value }\end{array}$ & $\mathrm{B}(95 \% \mathrm{Cl})$ & $\begin{array}{l}\mathrm{p}- \\
\text { value }\end{array}$ & B $(95 \% \mathrm{Cl})$ & $\begin{array}{l}\mathrm{p}- \\
\text { value }\end{array}$ \\
\hline \multicolumn{11}{|l|}{$\begin{array}{l}\text { Weekly } \\
\text { units of } \\
\text { alcohol }\end{array}$} \\
\hline 0 units & $\begin{array}{l}-2.48 \\
(-3.60 ;-1.36)\end{array}$ & $<.001$ & $\begin{array}{l}-1.89 \\
(-2.98 ;-0.81)\end{array}$ & 0.001 & $\begin{array}{l}-1.64 \\
(-2.72 ;-0.55)\end{array}$ & 0.003 & $\begin{array}{l}-1.64 \\
(-2.71 ;-0.57)\end{array}$ & 0.003 & $\begin{array}{l}-1.45 \\
(-2.50 ;-0.39)\end{array}$ & 0.007 \\
\hline $1-14$ units & Ref & - & Ref & - & Ref & - & Ref & - & Ref & - \\
\hline $\begin{array}{l}15-21 \\
\text { units }\end{array}$ & $\begin{array}{l}0.35 \\
(-0.89 ; 1.59)\end{array}$ & 0.577 & $\begin{array}{l}0.12 \\
(-1.07 ; 1.32)\end{array}$ & 0.838 & $\begin{array}{l}0.18 \\
(-1.00 ; 1.36)\end{array}$ & 0.766 & $\begin{array}{l}0.11 \\
(-1.06 ; 1.28)\end{array}$ & 0.851 & $\begin{array}{l}0.08 \\
(-1.07 ; 1.22)\end{array}$ & 0.897 \\
\hline$\geq 22$ units & $\begin{array}{l}-0.11 \\
(-1.54 ; 1.33)\end{array}$ & 0.885 & $\begin{array}{l}-0.24 \\
(-1.61 ; 1.14)\end{array}$ & 0.737 & $\begin{array}{l}-0.12 \\
(-1.49 ; 1.25)\end{array}$ & 0.862 & $\begin{array}{l}0.01 \\
(-1.37 ; 1.38)\end{array}$ & 0.992 & $\begin{array}{l}0.05 \\
(-1.29 ; 1.39)\end{array}$ & 0.941 \\
\hline \multicolumn{11}{|c|}{1 unit corresponds to 12 grams of pure alcohol } \\
\hline $\begin{array}{l}{ }^{a} \text { six obser } \\
\text { analyses ir }\end{array}$ & רs were exc & ed in & unadjusted & ses & eekly cons & on & en and five & rvat & $\mathrm{s}$ in the unac & \\
\hline
\end{tabular}


Table 4

FIML analyses on associations between alcohol consumption at baseline and SF-36 vitality score at follow-up in unadjusted and adjusted SEM models in men $(\mathrm{N}=3336)$ and women $(\mathrm{N}=812)$

\begin{tabular}{|c|c|c|c|c|c|c|c|c|c|c|}
\hline & \multicolumn{2}{|l|}{ Unadjusted $^{a}$} & \multicolumn{2}{|c|}{$\begin{array}{l}\text { Model } 1 \text { (age, cohort, } \\
\text { education, } \\
\text { employment) }\end{array}$} & \multicolumn{2}{|c|}{$\begin{array}{l}\text { Model } 1+\text { other } \\
\text { lifestyle factors }\end{array}$} & \multicolumn{2}{|c|}{$\begin{array}{l}\text { Model } 1+\text { social } \\
\text { relations }\end{array}$} & \multicolumn{2}{|c|}{ Model 1 + morbidity } \\
\hline $\begin{array}{l}\operatorname{Men}(N= \\
3336)\end{array}$ & $\mathrm{B}(95 \% \mathrm{Cl})$ & $\begin{array}{l}\mathrm{p}- \\
\text { value }\end{array}$ & $\mathrm{B}(95 \% \mathrm{Cl})$ & $\begin{array}{l}\mathrm{p}- \\
\text { value }\end{array}$ & $\mathrm{B}(95 \% \mathrm{Cl})$ & $\begin{array}{l}\mathrm{p}- \\
\text { value }\end{array}$ & $\mathrm{B}(95 \% \mathrm{Cl})$ & $\begin{array}{l}\mathrm{p}- \\
\text { value }\end{array}$ & B (95\% Cl) & $\begin{array}{l}\mathrm{p} \text { - } \\
\text { value }\end{array}$ \\
\hline \multicolumn{11}{|l|}{$\begin{array}{l}\text { Weekly } \\
\text { units of } \\
\text { alcohol } \\
\text { consumed }\end{array}$} \\
\hline 0 units & $\begin{array}{l}-8.06 \\
(-11.10 ;-5.03)\end{array}$ & $\begin{array}{l}<.001 \\
0.00\end{array}$ & $\begin{array}{l}-4.63 \\
(-7.61 ;-1.65)\end{array}$ & 0.002 & $\begin{array}{l}-3.09 \\
(-5.99 ;-0.18)\end{array}$ & 0.037 & $\begin{array}{l}-3.80 \\
(-6.77 ;-0.83)\end{array}$ & 0.012 & $\begin{array}{l}-2.20 \\
(-4.99 ; 0.59)\end{array}$ & 0.122 \\
\hline $\begin{array}{l}1-14 \\
\text { units }\end{array}$ & Ref & - & Ref & - & Ref & - & Ref & - & Ref & - \\
\hline $\begin{array}{l}15-21 \\
\text { units }\end{array}$ & $\begin{array}{l}0.86 \\
(-1.00 ; 2.72)\end{array}$ & 0.363 & $\begin{array}{l}0.73 \\
(-1.06 ; 2.53)\end{array}$ & 0.424 & $\begin{array}{l}0.76 \\
(-0.99 ; 2.51)\end{array}$ & 0.393 & $\begin{array}{l}0.66 \\
(-1.13 ; 2.45)\end{array}$ & 0.469 & $\begin{array}{l}0.79 \\
(-0.88 ; 2.46)\end{array}$ & 0.353 \\
\hline$\geq 22$ units & $\begin{array}{l}-2.77 \\
(-4.40 ;-1.14)\end{array}$ & 0.001 & $\begin{array}{l}-2.25 \\
(-3.83 ;-0.67)\end{array}$ & 0.005 & $\begin{array}{l}-0.90 \\
(-2.46 ; 0.65)\end{array}$ & 0.255 & $\begin{array}{l}-2.06 \\
(-3.63 ;-0.49)\end{array}$ & 0.010 & $\begin{array}{l}-1.52 \\
(-2.99 ;-0.06)\end{array}$ & 0.042 \\
\hline \multicolumn{11}{|l|}{$\begin{array}{l}\text { Binge } \\
\text { drinking } \\
\text { past } 30 \\
\text { days }^{b}\end{array}$} \\
\hline $\begin{array}{l}<\text { once per } \\
\text { month }\end{array}$ & $\begin{array}{l}-0.95 \\
(-2.80 ; 0.90)\end{array}$ & 0.315 & $\begin{array}{l}-0.21 \\
(-2.01 ; 1.58)\end{array}$ & 0.814 & $\begin{array}{l}-0.32 \\
(-2.06 ; 1.42)\end{array}$ & 0.719 & $\begin{array}{l}0.11 \\
(-1.67 ; 1.90)\end{array}$ & 0.900 & $\begin{array}{l}0.70 \\
(-0.98 ; 2.38)\end{array}$ & 0.413 \\
\hline $\begin{array}{l}1-3 \text { times } \\
\text { per month }\end{array}$ & Ref & - & Ref & - & Ref & - & Ref & - & Ref & - \\
\hline $\begin{array}{l}\geq \text { once } \\
\text { per week }\end{array}$ & $\begin{array}{l}-2.08 \\
(-3.83 ;-0.34)\end{array}$ & 0.019 & $\begin{array}{l}-1.47 \\
(-3.16 ; 0.22)\end{array}$ & 0.088 & $\begin{array}{l}-0.60 \\
(-2.24 ; 1.05)\end{array}$ & 0.478 & $\begin{array}{l}-1.22 \\
(-2.90 ; 0.47)\end{array}$ & 0.158 & $\begin{array}{l}-1.32 \\
(-2.90 ; 0.25)\end{array}$ & 0.100 \\
\hline $\begin{array}{l}\text { Women } \\
(\mathrm{N}=812)\end{array}$ & $\mathrm{B}(95 \% \mathrm{Cl})$ & $\begin{array}{l}\mathrm{p}- \\
\text { value }\end{array}$ & $\mathrm{B}(95 \% \mathrm{Cl})$ & $\begin{array}{l}\mathrm{p}- \\
\text { value }\end{array}$ & $\mathrm{B}(95 \% \mathrm{Cl})$ & $\begin{array}{l}\mathrm{p}- \\
\text { value }\end{array}$ & $\mathrm{B}(95 \% \mathrm{Cl})$ & $\begin{array}{l}\mathrm{p} \text { - } \\
\text { value }\end{array}$ & B (95\% Cl) & $\begin{array}{l}\mathrm{p}- \\
\text { value }\end{array}$ \\
\hline \multicolumn{11}{|l|}{$\begin{array}{l}\text { Weekly } \\
\text { units of } \\
\text { alcohol } \\
\text { consumed }\end{array}$} \\
\hline 0 units & $\begin{array}{l}-7.80 \\
(-11.94 ;-3.67)\end{array}$ & $\begin{array}{l}< \\
0.001\end{array}$ & $\begin{array}{l}-6.35 \\
(-10.44 ;-2.26)\end{array}$ & 0.002 & $\begin{array}{l}-5.25 \\
(-9.29 ;-1.21)\end{array}$ & 0.011 & $\begin{array}{l}-5.74 \\
(-9.80 ;-1.67)\end{array}$ & 0.006 & $\begin{array}{l}-4.11 \\
(-7.91 ;-0.31)\end{array}$ & 0.034 \\
\hline $\begin{array}{l}1-14 \\
\text { units }\end{array}$ & Ref & - & Ref & - & Ref & - & Ref & - & Ref & - \\
\hline $\begin{array}{l}15-21 \\
\text { units }\end{array}$ & $\begin{array}{l}3.20 \\
(-1.32 ; 7.71)\end{array}$ & 0.165 & $\begin{array}{l}2.50 \\
(-1.92 ; 6.92)\end{array}$ & 0.268 & $\begin{array}{l}2.45 \\
(-1.87 ; 6.78)\end{array}$ & 0.267 & $\begin{array}{l}2.42 \\
(-1.96 ; 6.80)\end{array}$ & 0.279 & $\begin{array}{l}2.11 \\
(-1.96 ; 6.19)\end{array}$ & 0.309 \\
\hline$\geq 22$ units & $\begin{array}{l}-0.30 \\
(-5.56 ; 4.97)\end{array}$ & 0.912 & $\begin{array}{l}-0.94 \\
(-6.09 ; 4.22)\end{array}$ & 0.722 & $\begin{array}{l}-0.52 \\
(-5.57 ; 4.52)\end{array}$ & 0.838 & $\begin{array}{l}-0.49 \\
(-5.68 ; 4.70)\end{array}$ & 0.853 & $\begin{array}{l}0.09 \\
(-4.70 ; 4.88)\end{array}$ & 0.970 \\
\hline \multicolumn{11}{|c|}{1 unit corresponds to 12 grams of pure alcohol } \\
\hline \multicolumn{11}{|c|}{$\begin{array}{l}\text { a ten observations were excluded in the unadjusted analyses on weekly consumption in men, seven observations in the unadjusted } \\
\text { analyses on binge drinking in men and five observations in the unadjusted analyses in women due to missing observations on all } \\
\text { variables }\end{array}$} \\
\hline
\end{tabular}


Approximately half of the participants had a weekly consumption of 1-14 units of alcohol (53.4\%), whereas few participants reported alcohol abstinence (7.0\%) (Table 1). The majority of participants were men (as the MP cohort only included men) and a tendency of women reporting lower alcohol consumption than men was observed. In Denmark, the high-risk drinking limits for men and women are 21 units and 14 units of alcohol per week, respectively, and $27 \%$ of the men and $17 \%$ of the women in the current study sample exceeded these limits. Concerning binge drinking, participants were almost equally distributed across the categories with binge drinking 'less than once a month' (26.7\%), '1-3 times per month' (41.1\%) and 'once per week or more' (32.2\%).

All investigated characteristics, except for age and rarely having someone to talk well with, were significantly associated with weekly alcohol consumption (Table 1). Individuals in the abstinent group were more likely to be characterized by low education, current unemployment, current smoking, sedentary leisure activities, BMI $\geq 30 \mathrm{~kg} / \mathrm{m}^{2}$, not having children, living alone, low self-rated health, and psychiatric and somatic morbidity. Moreover, individuals in the heavy alcohol consumption group ( $\geq 22$ units) were more likely to be characterized by current smoking and not having children.

Cross-sectional associations between alcohol consumption and vitality

Weekly units of alcohol consumed at baseline was significantly associated with vitality scores at baseline in both men ( $p<0.001)$ and women ( $p=0.004$ ) (Table 2 ). In both sexes, the lowest vitality score was observed in the abstinent group, whereas the highest score was observed in the group consuming 15-21 units per week. Binge drinking at baseline in men was also significantly associated with vitality at baseline ( $p=0.002)$; the highest vitality score was observed in men with binge drinking ' $1-3$ times per month', whereas the scores were fairly similar in men with binge drinking 'less than once per month' and 'once per week or more'.

Prospective associations between alcohol consumption and satisfaction with life

\section{Men}

Unadjusted analyses suggested that the SWLS score at follow-up was 3.3 points lower in men with alcohol abstinence at baseline (0 units/week; $p<0.001)$ and 1.0 points lower in men with heavy alcohol consumption at baseline $(\geq 22$ units/week; $p<0.001)$ than in men with moderate alcohol consumption (1-14 units/week) at baseline (Table 3). These differences attenuated after adjustment for sociodemographic factors, but the differences remained statistically significant. Similarly, differences attenuated but remained statistically significant after further adjustment.

With regard to binge drinking at baseline, the SWLS scores at follow-up were 1.0 points lower in men binge drinking 'less than once per month' $(p<0.001)$ and in men binge drinking 'once per week or more' $(p<0.001)$ compared with men binge drinking '1-3 times per month' (Table 3). These differences attenuated but remained statistically significant after adjustment for sociodemographic factors. The lower SWLS scores with binge drinking 'once per week or more' was robust to adjustment for further covariates.

\section{Women}

In women, unadjusted analyses suggested a 2.5 points lower SWLS score at follow-up in the abstinent group at baseline (0 units/week; $\mathrm{p}<$ 0.001) compared with the group consuming 1-14 units per week (Table 3). These differences attenuated but remained statistically significant after adjustment for sociodemographic factors and after further adjustment for other lifestyle factors, social relations, and morbidity.

Prospective associations between alcohol consumption and vitality

\section{Men}

Compared with men with moderate alcohol consumption (1-14 units/week) at baseline, unadjusted analyses suggested that the vitality score at follow-up was 8.1 points lower in men with alcohol abstinence ( 0 units/week; $\mathrm{p}<0.001)$ and 2.8 points lower in men with heavy alcohol consumption ( $\geq 22$ units/week; $p<0.001$ ) (Table 3). These differences attenuated after adjustment for sociodemographic factors but remained statistically significant. Nevertheless, differences became statistically non-significant after further adjustment for other lifestyle factors (only heavy drinking) and morbidity.

In relation to binge drinking at baseline, men with binge drinking 'once per week or more' had a 2.1 points lower vitality score at follow-up than men with binge drinking ' $1-3$ times per month' in the unadjusted analyses ( $p=0.019)$ (Table 4). Nevertheless, this difference became statistically non-significant after adjustment for sociodemographic factors and other covariates.

Page 10/15 


\section{Women}

In the unadjusted analyses, the vitality scores at follow-up were 7.8 points lower in women who were abstinent at baseline ( 0 units/week) compared with women who consumed 1-14 units per week $(p<0.001)$ (Table 4$)$. These differences attenuated but remained statistically significant after adjustment for sociodemographic factors and after further adjustment for other lifestyle factors, social relations, and morbidity.

\section{Discussion}

Main results

In this large study sample of Danish men and women, results suggested that alcohol abstinence compared with moderate alcohol consumption was associated with lower SWLS scores three to seven years later in both men and women even after adjustment for covariates. Moreover, men with heavy alcohol consumption at baseline had a lower SWLS score at follow-up than men with moderate alcohol consumption. Finally, men with moderate frequency of binge drinking ( 1 to 3 times per month) at baseline had higher SWLS scores at follow-up than men with higher frequency and men with lower frequency of binge drinking. Concerning vitality, results suggested that alcohol abstinence at baseline in both men and women and heavy alcohol consumption at baseline in men were associated with lower vitality scores at follow-up than moderate alcohol consumption.

Comparison with previous research

Our findings on alcohol consumption and satisfaction with life are partly consistent with previous findings from large prospective studies on middle-aged individuals and the general population. In relation to alcohol abstinence at baseline, Lang et al. [13] likewise observed lower subjective well-being three years later in this group compared with moderate alcohol consumption (up to two drinks/day) in middle-aged men and women. In addition, our findings of lower life satisfaction at follow-up with heavy alcohol consumption at baseline corroborate the findings of two large population-based studies including both men and women [9, 10]. Like in the present study, Massin and Kopp [10] only observed significant associations between heavy alcohol consumption at baseline and lower life satisfaction at follow-up in men, even though the analyses included a large number of women $(\mathrm{N}=3877)$. Finally, in relation to binge drinking, a previous population-based Finish study found that binge drinking at least once per month at baseline increased the risk of life dissatisfaction 15 years later [9]. This result is not consistent with our findings, which indicate an inverse u-shaped association with highest satisfaction with life in men with moderate frequency of binge drinking ( 1 to 3 times/month). Hence, our findings extend previous findings by suggesting that the highest satisfaction with life-at least in middle-aged Danish men-is observed with binge drinking one to three times per month.

In relation to alcohol consumption and vitality, our findings of lower vitality scores at follow-up in women with alcohol abstinence as opposed to moderate alcohol consumption at baseline are to some extent in line with previous findings. Schrieks et al. [14] observed that greater alcohol consumption (up to one daily serving) at baseline was associated with better health-related quality of life two years later in women aged 25-42 years from the Nurses' Health Study II. Like in the present study, the association was robust to adjustment for several covariates. Likewise, in a study on women aged 70 or older, Byles et al. [12] found that alcohol abstinence at baseline was associated with poorer health-related quality of life at follow-up. However, alcohol abstinence was only associated with the general health, physical functioning, mental health and social functioning subscales, and hence not with the vitality subscale. To our knowledge, no previous study that included men has observed a prospective association between alcohol abstinence and health-related quality of life. Hence, our findings of lower vitality scores at follow-up in men who were alcohol abstinent at baseline (findings that were not robust to adjustment for covariates) should be interpreted with caution. Finally, our results of lower vitality scores at follow-up with heavy alcohol consumption at baseline in men are in line with the findings from a previous male study [11]. In that study, alcohol consumption and health-related quality of life were assessed in 1974 and 2000, respectively, and only death-adjusted analyses suggested that men with heavy alcohol consumption ( $>349 \mathrm{~g} /$ week) at baseline had lower health-related quality of life at follow-up. The lack of robustness in the previous results as well as in our results (related to adjustment for lifestyle factors) could indicate that alcohol consumption is not central to health-related quality of life in men.

Interpretations

The observed prospective associations between alcohol consumption (weekly and binge drinking) and psychological well-being may be explained in several ways. First, alcohol may have a direct effect on psychological well-being. Since moderate consumption of alcohol is often associated with pleasure and positive effects such as stress reduction and letting go of control and heavy consumption is associated 
with negative effects such as intoxication and hangover, it is likely that alcohol affects psychological well-being directly in terms of higher psychological well-being in individuals with moderate alcohol consumption.

Second, several indirect pathways between alcohol consumption and psychological well-being may be anticipated. For example, alcohol consumption may influence the social relations of the individual that in turn influence psychological well-being. Due to the cultural acceptance and expectations of Danes to consume alcohol but also expectations about being in control of the alcohol consumption [33], it is likely that both alcohol abstinence and heavy alcohol consumption as well as infrequent and very frequent binge drinking adversely influence the social relationships of the individual; a factor that is highly central to psychological well-being [34, 35].

Third, it is also possible that underlying factors that influence both alcohol consumption and psychological well-being explain the observed associations. In particular in relation to the alcohol abstinent group-which in Denmark is a rather small group [36] with an alcohol behavior that fall outside the cultural norm [33]-several underlying factors may explain the observed lower psychological well-being. For example, some individuals may be non-drinkers due to illness or chronic diseases and even though we adjusted for comorbidity using Charlson Comorbidity Index, all diseases that potentially influence both alcohol consumption and psychological well-being are not necessarily included.

The observed sex differences in the association between heavy alcohol consumption at baseline and psychological well-being at follow-up could be explained by lack of power in the analyses among women, although sufficient power was present to observe cross-sectional associations among women. Another perhaps more plausible explanation-supported by data-is that the amount of alcohol consumed in the heavy alcohol consumption group is larger among men than among woman.

Our results suggested that alcohol consumption may be more strongly associated with satisfaction with life than vitality, since frequency of binge drinking was only prospectively associated with satisfaction with life and not with vitality, and larger standardized coefficients were observed in relation to satisfaction with life than vitality (data not shown). These observations could reflect that overall satisfaction with life over a three to seven-year period to a larger extent is influenced by the social consequences of alcohol consumption than the vitality of the individual, primarily reflecting current feelings of energy and fatigue.

Methodological considerations

The main advantage of the present study is the prospective design, large study sample including both men and women, inclusion of two alcohol consumption variables-average weekly consumption and binge drinking-as well as two aspects of psychological well-being in terms of both satisfaction with life and vitality. The study particularly strikes by addressing the knowledge gap on the association between alcohol consumption and satisfaction with life in middle-aged individuals and the association between binge drinking and psychological well-being in the same age group. Moreover, the study sample and included measures enabled us to investigate the association between alcohol consumption and psychological well-being according to sex, alcohol consumption pattern, and different aspects of psychological well-being. Finally, we had information on a broad range of possible confounding factors-including both self-reported and register-based information-which allowed to present the results adjusted for these factors.

The study also has some limitations. In relation to alcohol consumption, we did not have information on binge drinking in women, limiting the conclusions on alcohol consumption patterns to men. Moreover, underreporting of amount of alcohol consumed is a well-known problem [37] and alcohol consumption was assessed slightly different in the two included cohorts (past-week consumption in the MP cohort and usual consumption on weekdays and weekends in the DALWUH cohort). However, the latter is assumed to be a minor problem as cohort membership was included as a covariate in all the adjusted analyses. In relation to psychological well-being, only the vitality subscale from SF-36 was included in the assessment of participants, complicating comparison with previous studies that usually report results on the physical and mental component summary. Finally, even though we included several covariates in the analyses, residual confounding should still be considered. For example, unmeasured factors such as personality may influence both alcohol consumption and psychological well-being, and incomplete assessment of covariates may still confound the associations.

\section{Conclusions}

In conclusion, we found that alcohol abstinence was prospectively associated with lower psychological well-being compared with moderate alcohol consumption in both men and women. Additionally, heavy weekly alcohol consumption was prospectively associated with lower psychological well-being in men. With regard to binge drinking, an inverse u-shaped association was found in men between binge drinking at baseline and satisfaction with life (but not vitality) at follow-up. Thus, the results of the study suggest that middle-aged

Page $12 / 15$ 
Danes with a culturally 'normal' alcohol consumption have the highest psychological well-being several years later. The mechanisms behind these findings are, however, yet to be discovered and more research is warranted in this area.

\section{Declarations}

Ethics approval: According to Danish legislation, no ethics approval is needed for the present study. The study is covered by permissions from the Danish Data Protection Agency to the authors.

Consent to participate: Informed consent was obtained from all individual participants included in the study.

Consent for publication: Not applicable.

Availability of data and material: Data are not publicly available. To request access to the data from the Copenhagen Aging and Midlife Biobank, a formal application to the steering committee (Rikke Lund: rilo@sund.ku.dk) should be sent.

Competing interests: The authors declare that they have no conflicts of interest.

Funding: The IMK Almene Fond funded the current study through a grant to Trine Flensborg-Madsen. Moreover, all authors are part of the Phenotypes in Alcohol Use Disorders project, which is supported by Innovation Fund Denmark, Health and Clinical Research [grant number 603-00520B].

Authors' contributions: MG, CLW, ELM and TFM contributed to the study conception and design. Data analysis was performed by MG. The first draft of the manuscript was written by MG and CLW, ELM and TFM revised it critically for important intellectual content. MG, CLW, ELM and TFM approved the submitted version of the manuscript and have agreed both to be personally accountable for the author's own contributions and to ensure that questions related to the accuracy or integrity of any part of the work, even ones in which the author was not personally involved, are appropriately investigated, resolved, and the resolution documented in the literature..

Acknowledgements: The authors acknowledge the crucial role of the initiators and steering committees of the Metropolit Cohort and the Danish Longitudinal Study on Work Unemployment and Health, and they thank for their permission to conduct this study. Furthermore, the authors thank Kirsten Avlund, Helle Bruunsgaard, Nils-Erik Fiehn, Åse Marie Hansen, Poul Holm-Pedersen, Rikke Lund, Erik Lykke Mortensen, and Merete Osler who initiated and established the Copenhagen Aging and Midlife Biobank from 2009 to 2011. They also thank the staff at the Institute of Public Health and the National Research Center for the Working Environment who undertook the CAMB data collection.

\section{References}

1. Mäkelä, P., Raitasalo, K., \& Wahlbeck, K. (2015). Mental health and alcohol use: a cross-sectional study of the Finnish general population. European Journal of Public Health, 25(2), 225-231. https://doi.org/10.1093/eurpub/cku133

2. Okoro, C. A., Brewer, R. D., Naimi, T. S., Moriarty, D. G., Giles, W. H., \& Mokdad, A. H. (2004). Binge drinking and health-related quality of life: do popular perceptions match reality? American Journal of Preventive Medicine, 26(3), 230-233.

https://doi.org/10.1016/j.amepre.2003.10.022

3. Paul, L. A., Grubaugh, A. L., Frueh, B. C., Ellis, C., \& Egede, L. E. (2011). Associations between binge and heavy drinking and health behaviors in a nationally representative sample. Addictive Behaviors, 36(12), 1240-1245. https://doi.org/10.1016/j.addbeh.2011.07.034

4. Van Dijk, A. P., Toet, J., \& Verdurmen, J. E. E. (2004). The relationship between health-related quality of life and two measures of alcohol consumption. Journal of Studies on Alcohol, 65(2), 241-249. https://doi.org/10.15288/jsa.2004.65.241

5. Stranges, S., Notaro, J., Freudenheim, J. L., Calogero, R. M., Muti, P., Farinaro, E., ... Trevisan, M. (2006). Alcohol drinking pattern and subjective health in a population-based study. Addiction (Abingdon, England), 101(9), 1265-1276. https://doi.org/10.1111/j.13600443.2006.01517.x

6. Saarni, S. I., Joutsenniemi, K., Koskinen, S., Suvisaari, J., Pirkola, S., Sintonen, H., ... Lönnqvist, J. (2008). Alcohol consumption, abstaining, health utility, and quality of life--a general population survey in Finland. Alcohol and Alcoholism (Oxford, Oxfordshire), 43(3), 376-386. https://doi.org/10.1093/alcalc/agn003

Page $13 / 15$ 
7. Chan, A. M., von Mühlen, D., Kritz-Silverstein, D., \& Barrett-Connor, E. (2009). Regular alcohol consumption is associated with increasing quality of life and mood in older men and women: the Rancho Bernardo Study. Maturitas, 62(3), $294-300$. https://doi.org/10.1016/j.maturitas.2009.01.005

8. Valencia-Martín, J. L., Galán, I., Guallar-Castillón, P., \& Rodríguez-Artalejo, F. (2013). Alcohol drinking patterns and health-related quality of life reported in the Spanish adult population. Preventive Medicine, 57(5), 703-707. https://doi.org/10.1016/j.ypmed.2013.09.007

9. Koivumaa-Honkanen, H., Kaprio, J., Korhonen, T., Honkanen, R. J., Heikkilä, K., \& Koskenvuo, M. (2012). Self-reported life satisfaction and alcohol use: a 15-year follow-up of healthy adult twins. Alcohol and Alcoholism (Oxford, Oxfordshire), 47(2), $160-168$. https://doi.org/10.1093/alcalc/agr151

10. Massin, S., \& Kopp, P. (2014). Is life satisfaction hump-shaped with alcohol consumption? Evidence from Russian panel data. Addictive Behaviors, 39(4), 803-810. https://doi.org/10.1016/j.addbeh.2014.01.005

11. Strandberg, A. Y., Strandberg, T. E., Salomaa, V. V., Pitkälä, K., \& Miettinen, T. A. (2004). Alcohol consumption, 29-y total mortality, and quality of life in men in old age. The American Journal of Clinical Nutrition, 80(5), 1366-1371. https://doi.org/10.1093/ajen/80.5.1366

12. Byles, J., Young, A., Furuya, H., \& Parkinson, L. (2006). A drink to healthy aging: The association between older women's use of alcohol and their health-related quality of life. Journal of the American Geriatrics Society, 54(9), 1341-1347. https://doi.org/10.1111/j.1532-5415.2006.00837.x

13. Lang, I., Wallace, R. B., Huppert, F. A., \& Melzer, D. (2007). Moderate alcohol consumption in older adults is associated with better cognition and well-being than abstinence. Age and Ageing, 36(3), 256-261. https://doi.org/10.1093/ageing/afm001

14. Schrieks, I. C., Wei, M. Y., Rimm, E. B., Okereke, O. I., Kawachi, I., Hendriks, H. F. J., \& Mukamal, K. J. (2016). Bidirectional associations between alcohol consumption and health-related quality of life amongst young and middle-aged women. Journal of Internal Medicine, 279(4), 376-387. https://doi.org/10.1111/joim.12453

15. Mason, W. A., \& Spoth, R. L. (2011). Longitudinal associations of alcohol involvement with subjective well-being in adolescence and prediction to alcohol problems in early adulthood. Journal of Youth and Adolescence, 40(9), 1215-1224.

https://doi.org/10.1007/s10964-011-9632-z

16. Ortolá, R., García-Esquinas, E., Galán, I., \& Rodríguez-Artalejo, F. (2016). Patterns of alcohol consumption and health-related quality of life in older adults. Drug and Alcohol Dependence, 159, 166-173. https://doi.org/10.1016/j.drugalcdep.2015.12.012

17. Pisinger, C., Toft, U., Aadahl, M., Glümer, C., \& Jørgensen, T. (2009). The relationship between lifestyle and self-reported health in a general population: the Inter99 study. Preventive Medicine, 49(5), 418-423. https://doi.org/10.1016/j.ypmed.2009.08.011

18. Molnar, D. S., Busseri, M. A., Perrier, C. P. K., \& Sadava, S. W. (2009). A longitudinal examination of alcohol use and subjective wellbeing in an undergraduate sample. Journal of Studies on Alcohol and Drugs, 70(5), 704-713. https://doi.org/10.15288/jsad.2009.70.704

19. Cummins, R. A. (2008). The wellbeing of Australians: Links with exercise, nicotine and alcohol. Australian Unity Wellbeing Index Survey 19. Melbourne: Deakin University: Australian Centre on Quality of Life.

20. Avlund, K., Osler, M., Mortensen, E. L., Christensen, U., Bruunsgaard, H., Holm-Pedersen, P., ... Lund, R. (2014). Copenhagen Aging and Midlife Biobank (CAMB): An Introduction. Journal of Aging and Health, 26(1), 5-20. https://doi.org/10.1177/0898264313509277

21. Osler, M., Lund, R., Kriegbaum, M., Christensen, U., \& Andersen, A.-M. N. (2006). Cohort Profile: The Metropolit 1953 Danish Male Birth Cohort. International Journal of Epidemiology, 35(3), 541-545. https://doi.org/10.1093/ije/dyi300

22. Christensen, U., Lund, R., Damsgaard, M. T., Holstein, B. E., Ditlevsen, S., Diderichsen, F., ... Lynch, J. (2004). Cynical hostility, socioeconomic position, health behaviors, and symptom load: a cross-sectional analysis in a Danish population-based study. Psychosomatic Medicine, 66(4), 572-577. https://doi.org/10.1097/01.psy.0000126206.35683.d1

23. Lund, R., Mortensen, E. L., Christensen, U., Bruunsgaard, H., Holm-Pedersen, P., Fiehn, N.-E., ... Osler, M. (2016). Cohort Profile: The Copenhagen Aging and Midlife Biobank (CAMB). International Journal of Epidemiology, 45(4), 1044-1053.

https://doi.org/10.1093/ije/dyv149 
24. Diener, E., Emmons, R. A., Larsen, R. J., \& Griffin, S. (1985). The Satisfaction With Life Scale. Journal of Personality Assessment, 49(1), 71-75. https://doi.org/10.1207/s15327752jpa4901_13

25. Pavot, W., Diener, E., Colvin, C. R., \& Sandvik, E. (1991). Further validation of the Satisfaction with Life Scale: evidence for the crossmethod convergence of well-being measures. Journal of Personality Assessment, 57(1), 149-161.

https://doi.org/10.1207/s15327752jpa5701_17

26. Ware, J. E., \& Sherbourne, C. D. (1992). The MOS 36-item short-form health survey (SF-36). I. Conceptual framework and item selection. Medical Care, 30(6), 473-483.

27. Lins, L., \& Carvalho, F. M. (2016). SF-36 total score as a single measure of health-related quality of life: Scoping review. SAGE Open Medicine, 4. https://doi.org/10.1177/2050312116671725

28. Bjorner, J. B., Damsgaard, M. T., Watt, T., \& Groenvold, M. (1998). Tests of Data Quality, Scaling Assumptions, and Reliability of the Danish SF-36. Journal of Clinical Epidemiology, 51(11), 1001-1011. https://doi.org/10.1016/S0895-4356(98)00092-4

29. Bjorner, J. B., Thunedborg, K., Kristensen, T. S., Modvig, J., \& Bech, P. (1998). The Danish SF-36 Health Survey: Translation and Preliminary Validity Studies. Journal of Clinical Epidemiology, 51(11), 991-999. https://doi.org/10.1016/S0895-4356(98)00091-2

30. Lynge, E., Sandegaard, J. L., \& Rebolj, M. (2011). The Danish National Patient Register. Scandinavian Journal of Public Health, 39(7 Suppl), 30-33. https://doi.org/10.1177/1403494811401482

31. Charlson, M. E., Pompei, P., Ales, K. L., \& MacKenzie, C. R. (1987). A new method of classifying prognostic comorbidity in longitudinal studies: Development and validation. Journal of Chronic Diseases, 40(5), 373-383. https://doi.org/10.1016/00219681(87)90171-8

32. Quan, H., Li, B., Couris, C. M., Fushimi, K., Graham, P., Hider, P., ... Sundararajan, V. (2011). Updating and Validating the Charlson Comorbidity Index and Score for Risk Adjustment in Hospital Discharge Abstracts Using Data From 6 Countries. American Journal of Epidemiology, 173(6), 676-682. https://doi.org/10.1093/aje/kwq433

33. Grønkjær, M., Curtis, T., Crespigny, C. D., \& Delmar, C. (2011). Acceptance and expectance: Cultural norms for alcohol use in Denmark. International Journal of Qualitative Studies on Health and Well-being, 6(4). https://doi.org/10.3402/qhw.v6i4.8461

34. Amati, V., Meggiolaro, S., Rivellini, G., \& Zaccarin, S. (2018). Social relations and life satisfaction: the role of friends. Genus, 74(1). https://doi.org/10.1186/s41118-018-0032-z

35. Barger, S. D., Donoho, C. J., \& Wayment, H. A. (2009). The relative contributions of race/ethnicity, socioeconomic status, health, and social relationships to life satisfaction in the United States. Quality of Life Research, 18(2), 179-189. https://doi.org/10.1007/s11136008-9426-2

36. World Health Organization. (2018). Global status report on alcohol and health 2018. World Health Organization. Retrieved from https://apps.who.int/iris/handle/10665/274603

37. Davis, C. G., Thake, J., \& Vilhena, N. (2010). Social desirability biases in self-reported alcohol consumption and harms. Addictive Behaviors, 35(4), 302-311. https://doi.org/10.1016/j.addbeh.2009.11.001 